\title{
Mobility Lab to Assess Balance and Gait with Synchronized Body-worn Sensors
}

Martina Mancini ${ }^{1 *}$, Laurie King ${ }^{1}$, Arash Salarian'1, Lars Holmstrom², James McNames ${ }^{2}$ and Fay B Horak ${ }^{1,2}$

${ }^{1}$ Department of Neurology, School of Medicine, Oregon Health \& Science University, 505 NW 185th Avenue, Beaverton, OR 97006, USA ${ }^{2}$ APDM Inc, Portland, OR 97201 USA

\begin{abstract}
This paper is a commentary to introduce how rehabilitation professionals can use a new, body-worn sensor system to obtain objective measures of balance and gait. Current assessments of balance and gait in clinical rehabilitation are largely limited to subjective scales, simple stop-watch measures, or complex, expensive machines not practical or largely available. Although accelerometers and gyroscopes have been shown to accurately quantify many aspects of gait and balance kinematics, only recently a comprehensive, portable system has become available for clinicians. By measuring body motion during tests that clinicians are already performing, such as the Timed Up and Go test (TUG) and the Clinical Test of Sensory Integration for Balance (CITSIB), the additional time for assessment is minimal. By providing instant analysis of balance and gait and comparing a patient's performance to age-matched control values, therapists receive an objective, sensitive screening profile of balance and gait strategies. This motion screening profile can be used to identify mild abnormalities not obvious with traditional clinical testing, measure small changes due to rehabilitation, and design customized rehabilitation programs for each individual's specific balance and gait deficits.
\end{abstract}

\section{Need for Better Balance and Gait Assessments in Rehabilitation}

Reliable, sensitive, and clinically meaningful measures of balance and gait are critical for clinical assessment, as well as for studies of rehabilitation intervention, of patients with neurological disorders such as Parkinson's disease (PD), multiple sclerosis, stroke, and other conditions such as frailty and orthopedic disorders that lead to high fall risk [1]. In these patients, restoration of function may require a prolonged course of therapy that can be difficult to design because progress based on small improvements are difficult to clinically measure. Technology that quantifies balance and gait more sensitively and objectively than current clinical examinations could be very useful for rehabilitation.

However, functional capacity must be assessed in rehabilitation at various points in time so that the nature of the intervention (type, intensity, duration, frequency) can be re-evaluated periodically and modified as needed [2]. Moreover, rehabilitative care is delivered at various settings across the spectrum of an individual's care; including rehabilitation units, skilled nursing facilities, outpatient clinics, and the patient's home. If technology is going to be used at multiple settings, such technology must be portable, low cost, easy to use, and lightweight. Understanding the response to therapeutic interventions also requires feedback that enables the therapist to make better decisions to select the most effective therapies.

Rehabilitation of neurologic disease is steeped in a history of subjective observation. Comparatively, other areas of rehabilitation, such as cardiac rehabilitation, have more rapidly embraced advances in technology to document patient progress. For example, it is standard of care for a physical therapist to monitor heart rate and oxygen saturation during gait and these objective, physiologic measures indicate the cardiac rehabilitation status of the patient [3].

In neurologic rehabilitation, the challenges are different. Many times, neurologically impaired patients cannot accurately convey or may lack the insight or ability to tell the clinician about their progress. Practical and reliable clinical scales to assess balance and gait in these patients in a clinic environment have been limited to walking duration from 3 to $30 \mathrm{~m}$, distance walked in 2 to $12 \mathrm{~min}$ (stop-watch based), or clinical rating scales that are limited by clinicians bias, insensitivity to mild impairments (ceiling effects), and sometimes poor reliability [47]. These limitations are serious concerns for clinicians and researchers who want to monitor disease progression, determine intervention efficacy, or treat people with mild mobility deficits [8].

The value of a sophisticated gait laboratory or dynamic posturography in assessing even mild impairments [9-12] or effect of medications and rehabilitation interventions [13-17] on gait and balance is well-established. However, gait laboratories and dynamic posturography systems are expensive, require large dedicated spaces, dedicated personnel, proper installation and professional interpretation. These constraints make this type of sophisticated technology impractical for many clinical settings, precluding many clinicians from incorporating these measures into clinical decision making.

Recently, body-worn inertial sensors have been proposed as a portable, low-cost alternative to gait laboratories or dynamic posturography for measurements of gait and balance [18-23]. Inertial measures of gait and balance have been shown to be sensitive to mild mobility problems in patients with neuropathy, Parkinson's disease, and multiple sclerosis [24-27]. These results suggest that such measures may provide a sensitive means of measuring subtle mobility deficits in clinical settings. Despite the potential advantages of these systems in clinical practice, until recently they have not provided the necessary

${ }^{*}$ Corresponding author: Martina Mancini, PhD, Balance Disorder Lab Department of Neurology, OHSU 505 NW $185^{\text {th }}$ Avenue, Beaverton, OR, USA, Tel: 503-418-2604; Fax: 503-418-2701; E-mail: mancinim@ohsu.edu

Received November 11, 2011; Accepted December 12, 2011; Published December 12, 2011

Citation: Mancini M, King L, Salarian A, Holmstrom L, McNames J, et al. (2012) Mobility Lab to Assess Balance and Gait with Synchronized Body-worn Sensors. $J$ Bioengineer \& Biomedical Sci S1:007. doi:10.4172/2155-9538.S1-007

Copyright: (c) 2012 Mancini M, et al. This is an open-access article distributed under the terms of the Creative Commons Attribution License, which permits unrestricted use, distribution, and reproduction in any medium, provided the original author and source are credited. 
Citation: Mancini M, King L, Salarian A, Holmstrom L, McNames J, et al. (2012) Mobility Lab to Assess Balance and Gait with Synchronized Bodyworn Sensors. J Bioengineer \& Biomedical Sci S1:007. doi:10.4172/2155-9538.S1-007

guidance, simplicity, accuracy, or information needed to help guide therapy.

\section{Mobility Lab}

APDM's Mobility Lab ${ }^{\text {tix }}$ (Figure 1) (APDM Inc, http://apdm.com) is the first portable gait and balance laboratory designed for clinicians and clinical researchers. It was designed to streamline gait and balance assessment by making it easy to collect, store, analyze, and interpret data. Mobility Lab ${ }^{\text {TM }}$ (Figure 1) is composed of: 1) a set wireless, body-worn Opal ${ }^{\text {Ts }}$ inertial sensors, each with a docking station, 2) an Access Point for wireless data transmission and sub-millisecond synchronization of the independent sensors, 3) user-friendly software to guide the user and subject(s) through the testing protocols, and 4) automated analysis and reporting of the recorded data (Figure 4).

Depending on the specified test protocol, one to six Opals are attached to the body with Velcro straps (one on the low back for postural sway, two on the shanks for gait, one on the sternum for sit/ stand transitions, and two on the wrists for arm swing). Figure 2 shows a subject wearing the Opals while performing a 180-degree turn. While being guided through the protocol, the clinician can easily abort, repeat, or comment on trials as necessary. Mobility Lab ${ }^{\text {tu }}$ provides immediate access to measures of gait and balance along with matching control values to aid in research or clinical decision-making.

\section{Mobility lab plugins}

APDM offers a suite of plugins for Mobility Lab ${ }^{\text {mo }}$ that allows clinicians to customize their analytical software to satisfy their research, clinical or therapeutic needs. Each plugin is an instrumented version of a widely-accepted clinical protocol.

Instrumented Timed-up and go plugin (ITUG): Subjects are instructed to stand up from a chair, walk $7 \mathrm{~m}$, turn $180^{\circ}$, then walk back
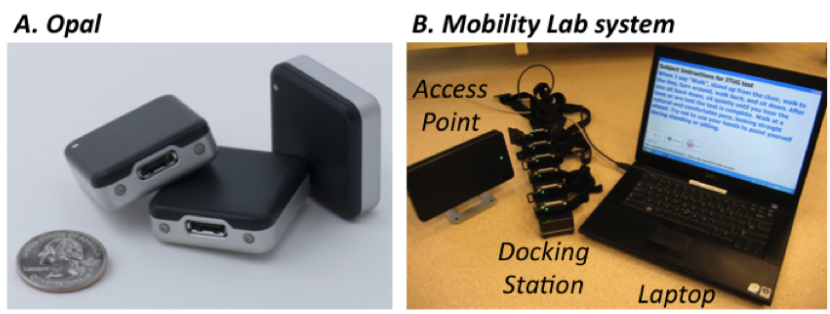

Figure 1: A. Sensor dimension. B. Mobility Lab system and components.

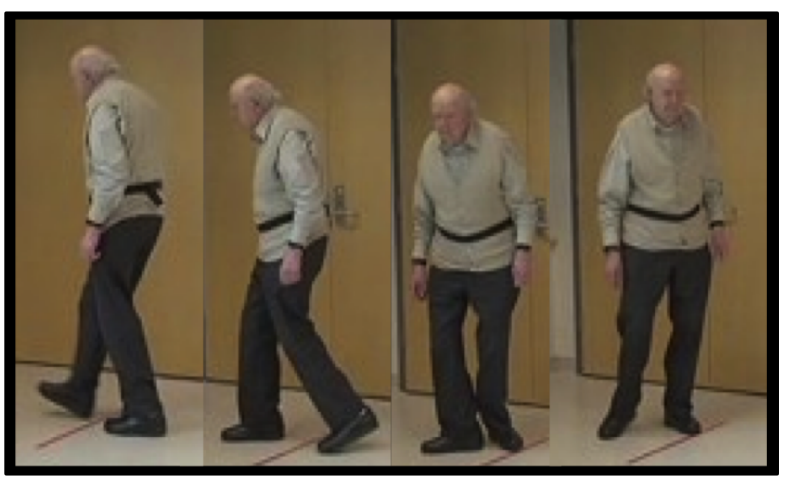

Figure 2: Five Opals applied to the body (ankles, wrists and lumbar) during the turning of the iTUG test. and sit down. The distance walked was extended from 3 to $7 \mathrm{~m}$ to allow processing of gait cycle data. The ITUG objectively characterizes 53 parameters during postural transitions (sit-to-stand and turn-to-sit), walking and turning [28]. Each of these parameters has been previously validated with a motion analysis system in a gait laboratory $[19,20]$.

Instrumented Sway (ISway): subjects are instructed to stand with arms at their side. The size of their stance is fixed with a spacer block placed between the feet. ISway objectively measures amplitude, velocity, frequency and jerkiness of postural sway in both the lateral and anterior-posterior directions with 42 metrics. Each of these metrics have been validated against postural sway measured from center of pressure displacement with a force place [27]. Therapists interested in performing the Clinical Test of Sensory Integration for Balance (CTSIB), measure sway while patients stand either on a firm surface or on compliant foam with eyes opened or closed to evaluate use of surface, visual, and vestibular information for postural sway $[18,29]$.

Instrumented Stand and Walk (ISAW): This short test was designed to combine measures of postural sway, anticipatory postural adjustments during step initiation, gait and turning into one, quick protocol. Subjects are instructed to stand quietly for 30 s and then asked to walk at their comfortable speed for $7 \mathrm{~m}$, turn $180^{\circ}$ and walk back to the starting point. Figure 3 summarizes the subcomponents of mobility and type of metrics calculated for the ISAW.

Instrumented Long Walk plugin (IWalk): This is the only plugin that doesn't have a fixed protocol since patients can walk any distance from $7 \mathrm{~m}$ up to $7 \mathrm{~km}$ as long as all of the walking is on a straight path and all of the turns are 180 degrees. IWalk allows additional gait parameters to be calculated that cannot otherwise be measured with short walking distances, such as gait variability, coordination (phase coordination index), and asymmetry [30,31].

\section{Mobility Lab Reporting}

Figure 4 shows examples of measures calculated in Mobility Lab from a PD patient undergoing deep brain stimulation (DBS). In this example, the ITUG and ISWAY were performed after six different stimulation settings. It is interesting to see that the $3^{\text {rd }}$ setting showed a worsening in balance and transitions but not in gait. In fact, sit-tostand duration and turn duration were the worst in the $3^{\text {rd }}$ DBS setting, while stride length and stride velocity are consistent across all settings. Also, sway jerk and sway frequency were the worst in that particular

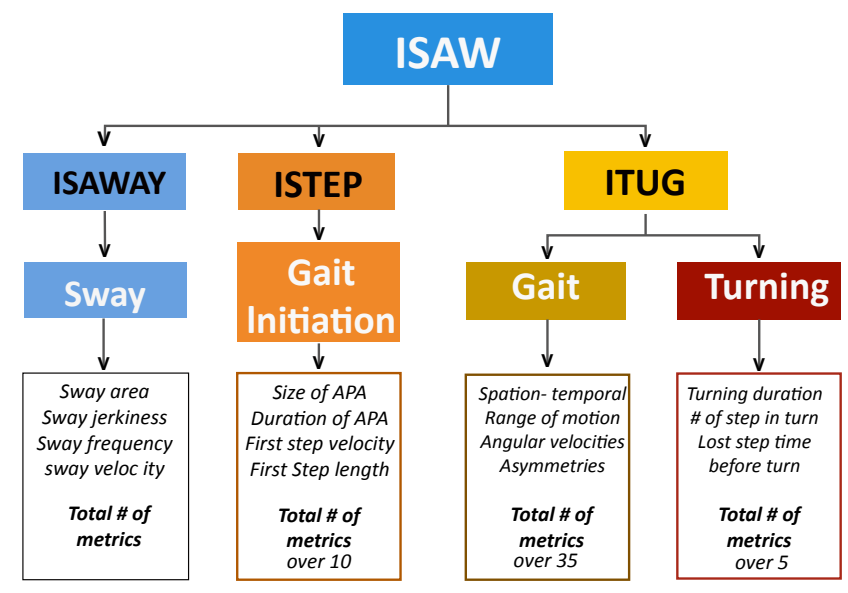

Figure 3: Components and metrics of ISAW, ITUG, ISWAY and ISTEP. 
Gait: Stride velocity

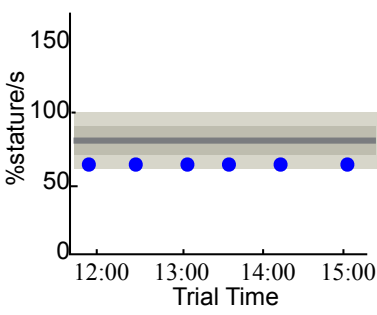

Turn: Duration

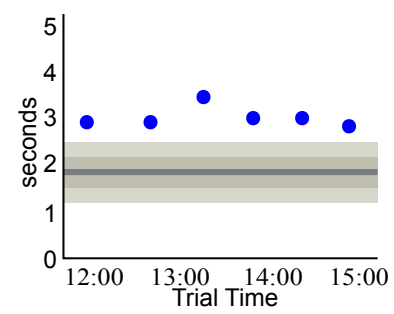

Normalized jerk

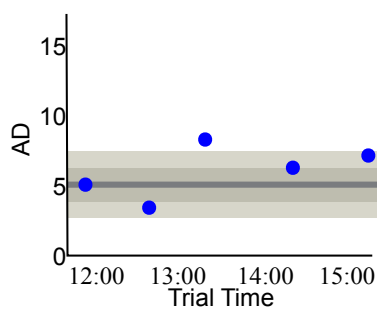

Gait: Stride length
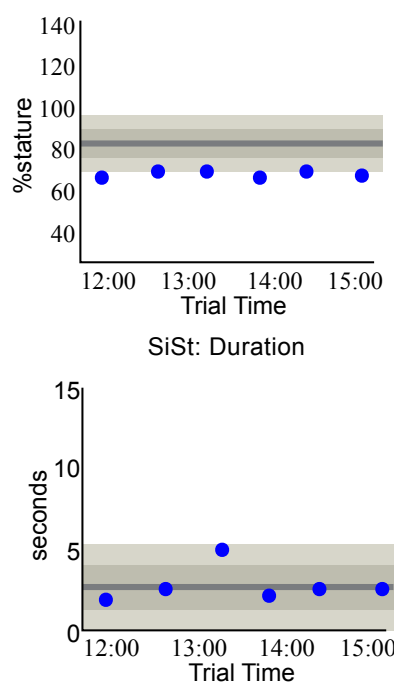

Median frequency

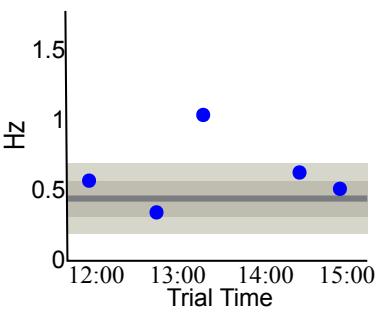

Figure 4: Example of the MobilityLab report.

Left panel: first page. Right panel: ISAW primary measures for 3 subsequen test in a PD patient, first test OFF Med OFF DBS, then OFF Med ON DBS, and ON Med ON DBS.
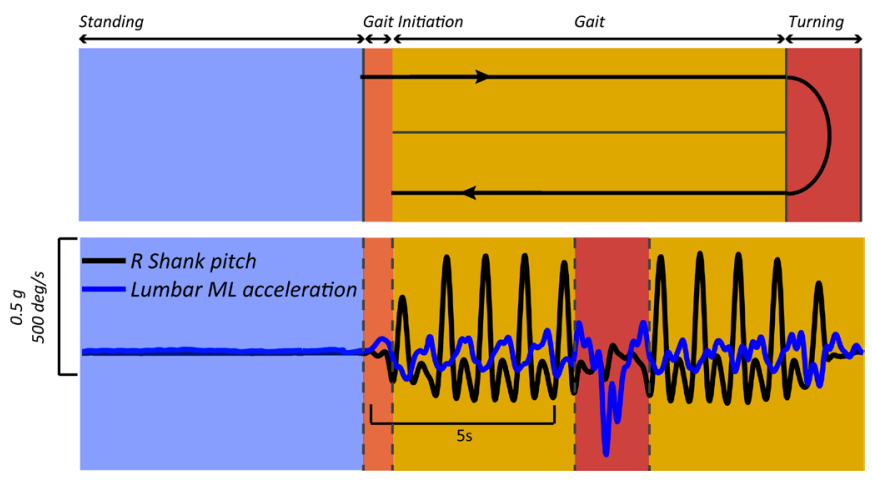

Figure 5: Raw signals from lower back and ankle sensors during ISAW.

setting. A patient with this particular DBS setting would need therapy for postural transitions and balance, but not for gait itself (Figure 4).

Figure 5 shows raw signals from the shank angular velocity and the medio-lateral trunk acceleration during standing, gait, and turning. From these raw signals, automatic analysis algorithms are developed to reliably quantify gait and balance parameters, validated by motion analysis and force plate gold-standard approaches in a laboratory (Figure 5).

Sensitivity, reliability, and validity of many of the plugins have been previously reported $[24,27,28,32,33]$. In particular, lateral postural sway, trunk rotation during gait, and arm swing during gait and turning are very sensitive to mild disease, such as mild-to-moderate untreated PD [24] and multiple sclerosis [25]. In fact, postural sway, anticipatory postural adjustments, arm speed, and turning velocity are sensitive measures in early PD patients who otherwise have normal Timed Up and Go times and gait speed [24,33,32]. Test-retest reliability, after taking the sensors off and re-applying them can be excellent (ICC ranged from .75 to .98), with help from algorithms that compensate for inconsistent sensor placement $[28,27]$. Concurrent validity of the ITUG and ISway have been established with popular clinical tests of balance and gait such as the posture and gait part (PIGD) of the UPDRS.

\section{Different Patients have Different Mobility Problems}

Assessment of balance abilities is important for accurate diagnosis, characterization of impairment, identification of fall risk, treatment planning, and evaluation of changes over time. This is difficult because balance control consists of many different underlying systems including stability during quiet stance, postural reactions to external disturbances, anticipatory postural adjustments, postural responses to perturbations and dynamic balance during gait $[34,8]$.

For these reasons, physical therapists need to identify specific impairments of balance control before selecting therapies to improve balance deficits. Customized therapy is more effective than general exercise $[34,29,35]$. Mobility Lab is an ideal instrument to objectively assess the different component of balance. Specifically, Mobility Lab measures stability during quiet stance with ISway (amplitude, frequency and fluidity of postural sway), dynamic balance during gait with ITUG and IWalk (trunk stability, percent of time in single and double support), and postural transitions with the sit-to-stand, turning measures, and anticipatory postural adjustments prior to step initiation.

\section{Responsiveness to Rehabilitation Intervention in PD}

Recently, we developed a sensorimotor-agility exercise program for prevention of mobility disability in PD [36]. In the present pilot study we investigated the effect of this sensorimotor-agility exercise

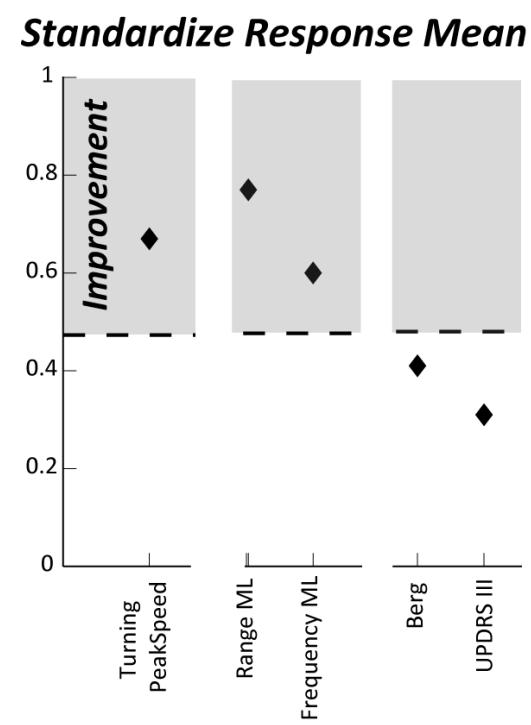

Figure 6: SRM of objective and clinical measures for the exercise study. 
Citation: Mancini M, King L, Salarian A, Holmstrom L, McNames J, et al. (2012) Mobility Lab to Assess Balance and Gait with Synchronized Bodyworn Sensors. J Bioengineer \& Biomedical Sci S1:007. doi:10.4172/2155-9538.S1-007

Page 4 of 5

program on different balance subcomponents in a group of patients with PD using Mobility Lab.

Twenty people with idiopathic PD (Age: $65.3 \pm 8$, UPDRS: 35.9 $\pm 15)$ participated in this delayed-start design study. Two baseline pretests (4 weeks apart) were obtained to determine stability of measures without intervention. Participants were under the direct supervision of a physical therapist, 4 times a week for 4 weeks, after which a posttest was administered. Subjects were tested in the ON medication state using UPDRS III Motor Part and the Berg Balance Scale.

Objective measures were obtained at each time point with ISway and ITUG.

In Figure 6, we show the Standardize Response Mean (SRM, [37]) for the principal outcomes. SRM is the mean change (d) reported in units of standard deviation of change (SDdiff), SRM=d/SDdiff . For SRM, a value of 0.20 represents a small change, of 0.50 a moderate, and a value of 0.80 represents a large change (Figure 6).

As we can see in Figure 6, the Berg Balance Scale and the UPDRSIII did not change after the exercise program. In contrast, the more sensitive objective measures provided by Mobility Lab revealed specific changes in medio-lateral sway and turning transitions after exercise.

\section{Specificity of Rehabilitation Intervention}

Each patient with a balance or gait problem requires a different rehabilitation intervention, depending on their primary functional constraints on balance control. Mobility Lab can assist in specifying

\section{A. Acceleration signals during quiet standing}

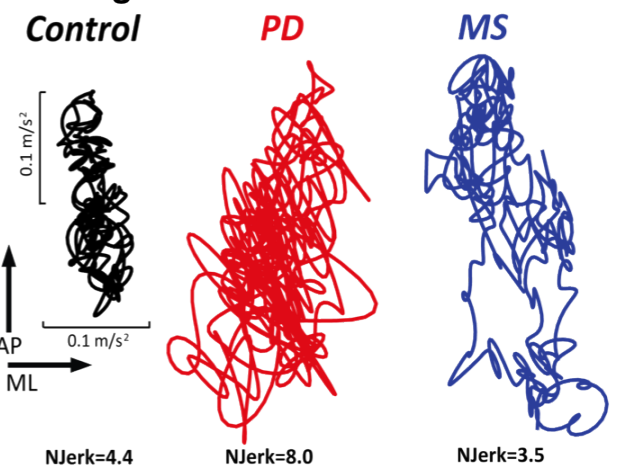

\section{B. Trunk Rotation Range of Motion} during gait

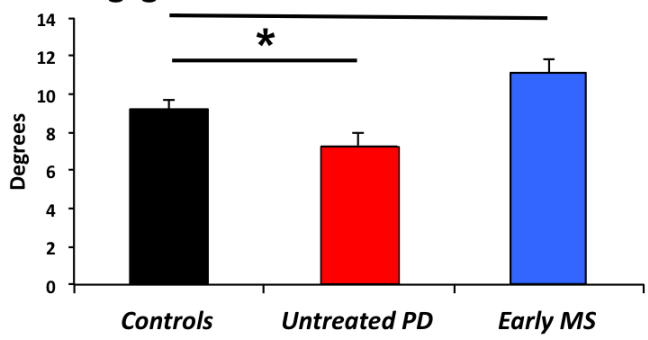

Figure 7: A. Acceleration signals recorded from the Lumbar sensor during 30 s of quietstance. Examplesin are presentative control, PD and MS subjects. the type of balance and/or deficit to therapy can be aimed at the problems. For example, Figure 7 illustrates postural sway during stance in a healthy control subject, a patient with PD and a patient with MS. Although both the PD and MS patients show larger postural sway area, the jerkiness of sway is increased in the PD patient but decreased in the MS patient. The increased jerk may reflect stiffness of the trunk and cocontraction of muscles for postural control in the PD subject whereas the decreased jerk may reflect slowed postural responses in the MS subject. In addition, a group of 12 untreated, early PD subjects showed decreased trunk rotation during gait whereas a group of 31 early MS subjects showed increased trunk rotation during gait, although there was no difference in gait speed among the groups [24,25]. Therapists with this type of assessment should focus on increasing trunk flexibility and rotation in the PD patient and increasing speed of postural response in the MS patient and they would expect very different changes due to intervention in the two subjects (Figure 7).

However, future studies are needed to determine reliability and validity in patients who are more disabled than those in the considered studies as well as those with a very different gait pattern, for example in post-stroke patients. In addition, although assessments performed in the clinical settings have value, it is often questioned whether assessments performed in such environment are truly representative of how a given clinical intervention affects the real life of the patients. To overcome this problem, either continuous recording of sensor data or at least monitoring over extended periods of time are necessary to better design and implement an effective clinical intervention.

\section{Conclusions}

Mobility Lab provides clinicians a fast, portable, and reliable system for quantifying balance and gait. Popular clinical tests, such as the Timed Up and Go (TUG) and the Clinical Test of Sensory Integration for Balance (CITSIB) are instrumented such that many objective measures of balance and gait are instantly available in the same amount of time it normally takes patients to perform these tests. Instead of simply measuring the duration of a trial, clinicians can now evaluate the strategy patients use to accomplish these tests and compare these results to gender-and age-matched control subjects. Characterization of balance and gait strategies, such as whether a patient shows high amplitude sway in the lateral direction or whether they increase their double support time during gait, can enable therapists to tailor their rehabilitation approach for individuals based on their specific deficiencies. Mobility Lab can be an important tool both for understanding and treating mobility disorders.

\section{Competing interests}

Drs. Salarian, Holmstrom, McNames and Horak have significant financial interests in APDM, a company that has a commercial interest in the results of this research and technology. This potential conflict of interest has been reviewed and managed by OHSU and the Integrity Oversight Council.

\section{References}

1. Rossier P, Wade DT (2001) Validity and reliability comparison of 4 mobility measures in patients presenting with neurologic impairment. Arch Phys Med Rehabil 82: 9-13.

2. Frontera W (2003) The importance of technology in rehabilitation. IEEE Eng Med Biol Mag 22: 25.

3. Binkley PF (2003) The next era of examination and management of the patient with cardiovascular disease. IEEE Eng Med Biol Mag 22: 23-24. 
Citation: Mancini M, King L, Salarian A, Holmstrom L, McNames J, et al. (2012) Mobility Lab to Assess Balance and Gait with Synchronized Bodyworn Sensors. J Bioengineer \& Biomedical Sci S1:007. doi:10.4172/2155-9538.S1-007

4. Ebersbach G, Baas H, Csoti I, Mungersdorf M, Deuschl G (2006) Scales in Parkinson's disease. J Neurol 4: 32-35.

5. Lord SE, Rochester L (2005) Measurement of community ambulation after stroke: current status and future developments. Stroke 36: 1457-1461.

6. Kaufman M, Moyer D, Norton J (2000) The significant change for the Timed 25foot Walk in the multiple sclerosis functional composite. Mult Scler 6: 286-290.

7. Haaxma CA, Bloem BR, Borm GF, Horstink MW (2008) Comparison of a timed motor test battery to the Unified Parkinson's Disease Rating Scale-III in Parkinson's disease. Mov Disord 23: 1707-1717.

8. Horak FB, Wrisley DM, Frank J (2009) The Balance Evaluation Systems Test (BESTest) to differentiate balance deficits. Phys Ther 89: 484-498.

9. Wurdeman SR, Huisinga JM, Filipi M, Stergiou N (2011) Multiple sclerosis affects the frequency content in the vertical ground reaction forces during walking. Clin Biomech (Bristol, Avon) 26: 207-212.

10. Nashner LM, Peters JF (1990) Dynamic posturography in the diagnosis and management of dizziness and balance disorders. Neurol Clin 8: 331-349.

11. Chastan N, Debono B, Maltete D, Weber J (2008) Discordance between measured postural instability and absence of clinical symptoms in Parkinson's disease patients in the early stages of the disease. Mov Disord 23: 366-372.

12. Carpinella I, Crenna P, Calabrese E, Rabuffetti M, Mazzoleni P, et al. (2007) Locomotor function in the early stage of Parkinson's disease. IEEE Trans Neural Syst Rehabil Eng 15: 543-551.

13. Zijlstra A, Mancini M, Chiari L, Zijlstra W (2010) Biofeedback for training balance and mobility tasks in older populations: a systematic review. J Neuroeng Rehabil 7: 58.

14. Rocchi L, Chiari L, Horak FB (2002) Effects of deep brain stimulation and levodopa on postural sway in Parkinson's disease. J Neurol Neurosurg Psychiatry 73: 267-274

15. Huisinga JM, Filipi ML, Stergiou N (2011) Elliptical exercise improves fatigue ratings and quality of life in patients with multiple sclerosis. J Rehabil Res Dev 48: $881-890$.

16. Carpinella I, Crenna P, Marzegan A, Rabuffetti M, Rizzone M, et al. (2007) Effect of L-dopa and subthalamic nucleus stimulation on arm and leg swing during gait in Parkinson's Disease. Conf Proc IEEE Eng Med Biol Soc 2007: $6665-6668$.

17. Beuter A, Hernandez R, Rigal R, Modolo J, Blanchet PJ (2008) Postural sway and effect of levodopa in early Parkinson's disease. Can J Neurol Sci 35: 65-68.

18. Whitney SL, Roche JL, Marchetti GF, Lin CC, Steed DP, et al. (2011) A comparison of accelerometry and center of pressure measures during computerized dynamic posturography: a measure of balance. Gait Posture 33 594-599.

19. Salarian A, Russmann H, Vingerhoets FJ, Dehollain C, Blanc Y, et al. (2004) Gait assessment in Parkinson's disease: toward an ambulatory system for long-term monitoring. IEEE Trans Biomed Eng 51: 1434-1443.

20. Salarian A, Russmann H, Vingerhoets FJ, Burkhard PR, Aminian K (2007) Ambulatory monitoring of physical activities in patients with Parkinson's disease. IEEE Trans Biomed Eng 54: 2296-2299.
21. Moe-Nilssen R, Helbostad JL (2002) Trunk accelerometry as a measure of balance control during quiet standing. Gait Posture 16: 60-68.

22. Moe-Nilssen R (1998) Test-retest reliability of trunk accelerometry during standing and walking. Arch Phys Med Rehabil 79: 1377-1385.

23. Mayagoitia RE, Lotters JC, Veltink PH, Hermens H (2002) Standing balance evaluation using a triaxial accelerometer. Gait Posture 16: 55-59.

24. Zampieri C, Salarian A, Carlson-Kuhta P, Aminian K, Nutt JG, et al. (2010) The instrumented timed up and go test: potential outcome measure for disease modifying therapies in Parkinson's disease. J Neurol Neurosurg Psychiatry 81 : 171-176.

25. Spain R, StGeorge RJ, Salarian A, Mancini M, Wagner JM, et al. (2011) Bodyworn motion sensors detect balance and gait deficits in people with multiple sclerosis whohave normal walking speed. Gait Posture in press.

26. Najafi B, Horn D, Marclay S, Crews RT, Wu S, et al.(2010) Assessing postura control and postural control strategy in diabetes patients using innovative and wearable technology. J Diabetes Sci Technol 4 : 780-791.

27. Mancini M, Salarian A, Carlson-Kuhta P, Zampieri C, King L, et al. (2011) iSWAY: a Sensitive, Valid and Reliable Measure of Postural Control. J Neuroeng Rehabil Under Review.

28. Salarian A, Horak FB, Zampieri C, Carlson-Kuhta P, Nutt JG, Aminian K (2010) iTUG, a sensitive and reliable measure of mobility. IEEE Trans Neural Sys Rehabil Eng 18: 303-310.

29. Shumway-Cook A, Horak FB (1986) Assessing the influence of sensory interaction of balance. Suggestion from the field. Phys Ther $66: 1548-1550$.

30. Sant'Anna A, Salarian A, Wickstrom N (2011) A new measure of movement symmetry in early Parkinson's disease patients using symbolic processing of inertial sensor data. IEEE Trans Biomed Eng 58 : 2127-2135.

31. Plotnik M, Giladi N, Hausdorff JM (2007) A new measure for quantifying the bilateral coordination of human gait: effects of aging and Parkinson's disease. Exp Brain Res 181: 561-570.

32. Mancini M, Horak FB, Zampieri C, Carlson-Kuhta P, Nutt JG, et al. (2011) Trunk accelerometry reveals postural instability in untreated Parkinson's disease. Parkinsonism Relat Disord 17 : 557-562.

33. Mancini M, Zampieri C, Carlson-Kuhta P, Chiari L, Horak FB (2009) Anticipatory postural adjustments prior to step initiation are hypometric in untreated Parkinson's disease: an accelerometer-based approach. Eur J Neurol 16 : 1028-1034.

34. Sibley KM, Straus SE, Inness EL, Salbach NM, Jaglal SB (2011) Balance assessment practices and use of standardized balance measures among ontario physical therapists. Phys Ther $91: 1583-1591$.

35. Horak FB, King LA (2010) Invited commentary. Phys Ther 90 : 491-492.

36. King LA, Horak FB (2009) Delaying mobility disability in people with Parkinson disease using a sensorimotor agility exercise program. Phys Ther 89 : 384-393.

37. Moe-Nilssen R, Nordin E, Lundin-Olsson L (2008) Criteria for evaluation of measurement properties of clinical balance measures for use in fall prevention studies. Journal of evaluation in clinical practice $14: 236-240$.

This article was originally published in a special issue, Emerging Technology for Use in Rehabilitation handled by Editor(s). Dr. Philip Rowe, "University of Strathclyde", UK. 